\title{
Medizin und Mobilität
}

Flugmedizin Tropenmedizin Reisemedizin und etwas kleiner geschrieben Tauchmedizin Bergmedizin Expeditionsmedizin - unsere Zeitschrift spiegelt das wider, was auch das Konzept der Tagung Medizin und Mobilität ist: Gemeinsam ein Forum etablieren, das Wissen über die „Medizin unterwegs“ schafft. In Zukunft wird das Journal Fort- und Weiterbildung zu Lasten von Reiseberichten noch weiter ausbauen. Auch Sie sind aufgerufen, eigene Fallbeispiele einzureichen und zu diskutieren, damit das gemeinsame Journal noch mehr lebt und Sie selbst es dazu nutzen können, anderen wichtige Dinge mitzuteilen.

Vielleicht sollte man langsam anfangen daran zu denken, einen gemeinsamen Dachverband zu gründen, zu dem sich alle die Zeitschrift tragenden Gesellschaften zusammentun. Dieser hätte dann mehr Gewicht als Einzelgruppierungen, wenn wichtige Entscheidungen auf dem Gebiet „Medizin und Mobilität“ - wie jetzt die Neuregelung der G-35-Untersuchungen getroffen werden. Dies würde natürlich bedeuten, dass man sich in kritischen Fragen im Vorfeld einigen müsste, was zwar nicht immer leicht sein dürfte, aber am Ende doch zu besseren Ergebnissen führen würde.

Für den Kongress Medizin und Mobilität durften wir in diesem Jahr die tollen Tagungsräume der IHK Köln nutzen. Dies hat sicher sehr dazu beigetragen, dass der Kongress so gut angekommen ist - wir konnten diesmal exakt 300 Teilnehmer begrüßen. Endlich ist es auch gelungen, die Industrie mit ihren Ständen so einzubeziehen, dass sie mitten im Geschehen war und eine Vielzahl von Kontakten knüpfen konnte. Das beim Kongressdinner gezündete tolle „Privatfeuerwerk“ war ein krönender Abschluss. Abschluss deshalb, weil sich das Insti- tut für Luft- und Raumfahrtmedizin leider von der Organisation dieses Kongresses verabschieden muss. Aufgrund einer Vielzahl (sehr positiver!) Entwicklungen wird uns diese viele Ressourcen verbrauchende Kongressorganisation in Zukunft nicht mehr möglich sein. Ich hoffe, die DGLRM, die in Zukunft den Kongress alle zwei Jahre zu organisieren plant, führt das Konzept weiter zum Erfolg. In Zukunft wird mein Institut - dann allerdings als Teilnehmer natürlich weiter diesen Kongress unterstützen. Unsere gemeinsame Zeitung brauchen wir jetzt umso mehr. Das Editorial Board wird zusammen mit dem Georg Thieme Verlag darauf achten, ein breites Themenspektrum mit qualitativ hochwertigem Inhalt anzubieten. Je mehr Sie aktiv mitarbeiten und auch Kritik üben und Verbesserungsvorschläge machen (z. B. an die Redaktion, siehe Impressum), desto mehr wird das „unsere“ Zeitschrift. Deshalb: Seien Sie aktiv und bringen Sie sich ein!

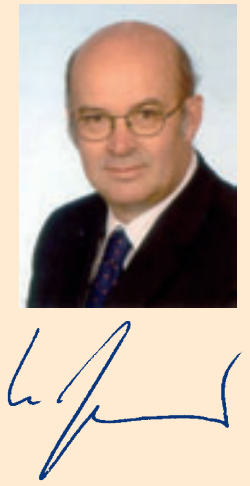

Prof. Dr. Rupert Gerzer,

Köln 